\title{
Collisionless momentum transfer in space and astrophysical explosions
}

\author{
A. S. Bondarenko ${ }^{1 \star}$, D. B. Schaeffer ${ }^{1}$, E. T. Everson1, S. E. Clark¹, B. R. Lee ${ }^{2}$, C. G. Constantin , \\ S. Vincena ${ }^{1}$, B. Van Compernolle ${ }^{1}$, S. K. P. Tripathi ${ }^{1}$, D. Winske ${ }^{3}$ and C. Niemann ${ }^{1}$
}

The AMPTE (Active Magnetospheric Particle Tracer Explorers) mission provided in situ measurements of collisionless momentum and energy exchange between an artificial, photo-ionized barium plasma cloud and the streaming, magnetized hydrogen plasma of the solar wind ${ }^{1-3}$. One of its most significant findings was the unanticipated displacement of the barium ion 'comet head' (and an oppositely directed deflection of the streaming hydrogen ions) transverse to both the solar wind flow and the interplanetary magnetic field, defying the conventional expectation that the barium ions would simply move downwind ${ }^{4}$. While subsequent theoretical and computational efforts $^{5-7}$ to understand the cause of the transverse motion reached differing conclusions, several authors ${ }^{5}$ attributed the observations to Larmor coupling 8,9 , a collisionless momentum exchange mechanism believed to occur in various astrophysical and space-plasma environments ${ }^{10,11}$ and to participate in cosmic magnetized collisionless shock formation ${ }^{12-14}$. Here we present the detection of Larmor coupling in a reproducible laboratory experiment that combines an explosive laser-produced plasma cloud with preformed, magnetized ambient plasma in a parameter regime relevant to the AMPTE barium releases. In our experiment, time-resolved Doppler spectroscopy reveals ambient ion acceleration transverse to both the laser-produced plasma flow and the background magnetic field. Utilizing a detailed numerical simulation, we demonstrate that the ambient ion velocity distribution corresponding to the measured Doppler-shifted spectrum is qualitatively and quantitatively consistent with Larmor coupling.

The AMPTE barium release experiments aimed to better understand a ubiquitous category of astrophysical and space phenomena characterized by the rapid expansion or relative motion of a dense 'debris' plasma cloud through relatively tenuous, magnetized, ambient plasma. Examples include the expansion of stellar material through the surrounding interstellar medium in supernova remnants ${ }^{10}$, the formation of cometary plasma tails due to the solar wind ${ }^{15}$, the interaction of interplanetary coronal mass ejections with the Earth's magnetosphere ${ }^{16}$, and man-made ionospheric explosions ${ }^{17}$. In these rarified environments, the Coulomb collisional mean free paths exceed the observed interaction length scales by many orders of magnitude, signifying that the debris plasma exchanges momentum and energy with the ambient plasma via collisionless, collective, electromagnetic effects. In addition, the relative motion of the debris cloud produces electric polarization fields between the magnetically confined electrons and the relatively freestreaming ions, resulting in $\mathbf{E} \times \mathbf{B}$ drift electron currents that expel the magnetic field within the cloud volume (the diamagnetic cavity) and enhance it at the cloud edge (the magnetic compression $)^{18}$. The general evolution in the reference frame of the magnetized ambient plasma is thus a deceleration of the debris cloud as it couples to the ambient plasma via collisionless processes and deforms the magnetic field.

We here consider explosive astrophysical and space environments characterized by a perpendicular-to-B debris plasma flow speed $V_{\mathrm{d}}$ that exceeds the ambient plasma Alfvén speed $v_{\mathrm{A}}$ (Alfvénic Mach number $M_{\mathrm{A} \perp} \equiv V_{\mathrm{d}} / v_{\mathrm{A}}>1$ ) and a magnetic pressure $B^{2} / 8 \pi$ that dominates the electron thermal pressure $p_{\mathrm{e}}$ (electron beta $\left.\beta_{\mathrm{e}} \equiv 8 \pi p_{\mathrm{e}} / B^{2} \ll 1\right)$. In this parameter regime, collisionless momentum and energy transfer due to plasma turbulence is generally ineffective, as the onset of candidate instabilities requires the condition $M_{\mathrm{A} \perp} \lesssim\left(1+\beta_{\mathrm{e}}\right)^{1 / 2}$ to be met ${ }^{10,19,20}$. Consequently, coupling is predominantly attributed to large-scale polarization and induction (or 'laminar') electric fields. In the framework of a 'hybrid' model, which treats the various ion species kinetically and the electrons as a single charge-neutralizing inertia-less fluid, a general expression for the laminar electric field follows from a combination of the electron fluid momentum equation with Ampere's law in the low-frequency (Darwin) limit:

$$
\mathbf{E}_{\mathrm{lam}}=-\frac{\mathbf{B} \times(\nabla \times \mathbf{B})}{4 \pi e \sum_{i} Z_{i} n_{i}}-\frac{\sum_{i} Z_{i} n_{i} \mathbf{v}_{i} \times \mathbf{B}}{c \sum_{i} Z_{i} n_{i}}
$$

Equation (1) gives the self-consistent electric field structure in terms of the charge numbers $Z_{i}$, densities $n_{i}$ and velocities $\mathbf{v}_{i}$ of all the ion species, the magnetic field profile $\mathbf{B}$, the elementary charge $e$, and the speed of light $c$ (see Supplementary Section I for a derivation). The first term of equation (1) indicates that an electric field must exist at the magnetic compression front and diamagnetic cavity edge (where the magnetic field is spatially non-uniform), while the second term denotes an electric field in regions containing ion currents moving across the magnetic field. In the super-Alfvénic limit $\left(M_{\mathrm{A} \perp} \gg 1\right)$, the second term becomes dominant ${ }^{21}$ and the laminar electric field in the reference frame of the ambient plasma points in the direction of $-\sum_{i} Z_{i} n_{i} \mathbf{v}_{i} \times \mathbf{B}$, transverse to the ion currents associated with the moving debris cloud and to the magnetic field. The corresponding kinetic response of stationary ambient ions is an initial acceleration in the direction of the transverse electric field and a subsequent deflection due to the magnetic field, in accordance with the Lorentz force. Within a time interval on the order of the ambient ion gyroperiod, the ions are 'rotated' by the magnetic field into the original direction of the debris flow. This interaction, termed Larmor coupling, thus allows the moving debris cloud to pick up the swept-over ambient ions.

\footnotetext{
${ }^{1}$ Department of Physics and Astronomy, University of California - Los Angeles, Los Angeles, California 90095, USA. ${ }^{2}$ Institut für Kernphysik, Technische Universität Darmstadt, 64289 Darmstadt, Germany. ${ }^{3}$ Los Alamos National Laboratory, Los Alamos, New Mexico 87545, USA. *e-mail: AntonBondarenko@ymail.com
} 


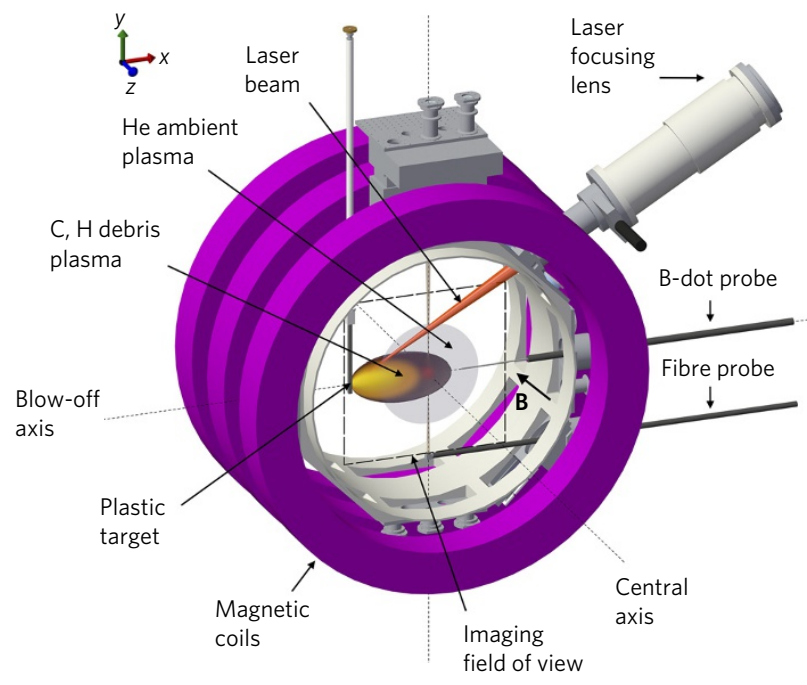

b

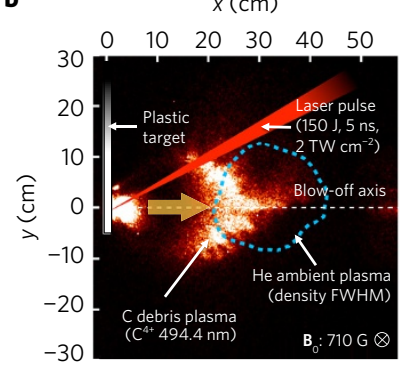

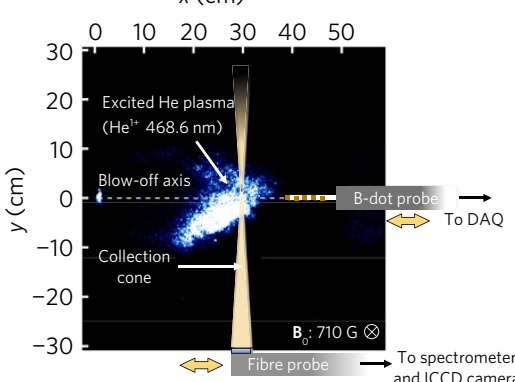

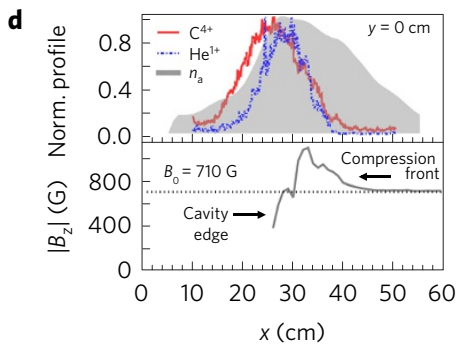

Figure 1 | Overview of the experiment. The LAPD creates reproducible, steady-state He plasma via a pulsed cathode-anode discharge, and an array of magnetic coils provides an axial magnetic field of $710 \mathrm{G}$ (directed in $-z$ ) that radially confines the plasma. The magnetized plasma column, which is approximately aligned to the central axis of the LAPD, has a peak electron density and temperature of $7.2 \times 10^{12} \mathrm{~cm}^{-3}$ and 4.3 eV, a He $\mathrm{e}^{1+}$ ion temperature of $\leq 0.5 \mathrm{eV}$, and a diameter of approximately $20 \mathrm{~cm}$ (based on density full-width at half-maximum). The Raptor laser, operating at $150 \pm 20 \mathrm{~J}$ per $5 \mathrm{~ns}$ pulse at a wavelength of $1,053 \mathrm{~nm}$, is focused to an intensity of approximately $2 \mathrm{TW} \mathrm{cm}^{-2}$ onto the surface of a rectangular high-density polyethylene (HDPE) plastic target embedded $30 \mathrm{~cm}$ from the central axis, producing an explosive $\mathrm{C}$ and $\mathrm{H}$ debris plasma cloud consisting of $\sim 10{ }^{17}$ ions. The debris expands

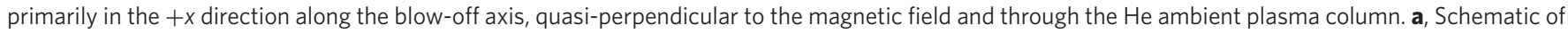
the LAPD section utilized for the experiment. A spectroscopic fibre probe, a magnetic flux (or 'B-dot') probe, and a fast wavelength-filtered camera (only imaging field of view is shown) monitor the debris-ambient interaction. b,c, Illustrations of the blow-off plane (the central $x-y$ plane containing the target) superimposed on filtered images of the expanding debris plasma (via $\mathrm{C}^{4+}$ ion emission at $494.4 \mathrm{~nm}$ ) and the excited ambient plasma (via He $\mathrm{H}^{1+}$ ion emission at $468.6 \mathrm{~nm}$ ) taken $750 \mathrm{~ns}$ after the laser pulse and integrated over $30 \mathrm{~ns}$. The fibre probe collects line-integrated emission through the excited $\mathrm{He}^{1+}$ ions along $y$ (perpendicular to both the blow-off axis and the magnetic field) at various distances from the target and is coupled to a spectrometer and intensified charge-coupled device (ICCD) camera that resolve the $\mathrm{He}^{1+} 468.6 \mathrm{~nm}$ spectral line to approximately $0.02 \mathrm{~nm}$. The time-integrated signal

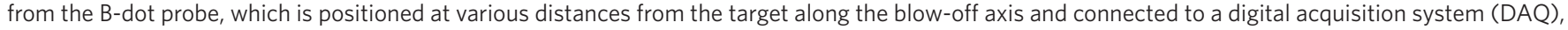

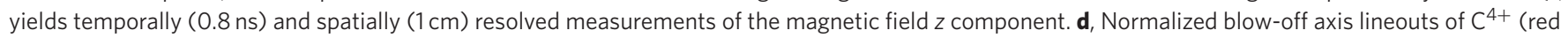
solid line) and $\mathrm{He}^{1+}$ (dashed blue line) emission from the filtered images of $\mathbf{b}$ and $\mathbf{c}$ compared to the ambient electron density profile (grey fill) and magnetic field z component magnitude (grey line), showing the correspondence between the magnetic compression front and emission leading edge.

Larmor coupling has received extensive theoretical and numerical investigation over the past few decades, both as a basic plasma process $^{8,9}$ and as a key mechanism in the evolution of various astrophysical and space phenomena, including man-made ionospheric explosions ${ }^{11}$, the AMPTE artificial comets ${ }^{5}$, and the formation of cosmic magnetized collisionless shocks ${ }^{12-14}$. However, this process has never before been observed in a laboratory setting. Despite numerous experiments utilizing laser-produced plasmas to simulate space and astrophysical explosions ${ }^{22}$, including noteworthy recent studies that successfully generated magnetized collisionless shocks ${ }^{23,24}$ and measured the electrostatic component of the electric field structure associated with an explosive plasma cloud ${ }^{25,26}$, none of these efforts examined the ion dynamics in sufficient detail to confirm Larmor coupling as a participating momentum exchange mechanism.

Here we present the first detection of Larmor coupling in a reproducible laboratory environment. In our experiment, detailed in Fig. 1, a plastic target embedded in the Large Plasma Device (LAPD $)^{27}$ is irradiated by the Raptor high-energy laser ${ }^{28}$, producing an explosive carbon $(\mathrm{C})$ and hydrogen $(\mathrm{H})$ debris plasma that expands quasi-perpendicular to the magnetic field and through the steady-state, magnetized helium (He) plasma column generated by the LAPD. Under the experimental parameters, the initial perpendicular-to-B debris expansion speed of $V_{\mathrm{d}} \approx 600 \mathrm{~km} \mathrm{~s}^{-1}$ (inferred from the magnetic compression front time-of-flight) exceeds the ambient plasma Alfvén speed of $v_{\mathrm{A}} \approx 290 \mathrm{~km} \mathrm{~s}^{-1}$, indicating a super-Alfvénic flow with $M_{\mathrm{A} \perp} \approx 2$. In addition, magnetic pressure dominates electron thermal pressure in the debris-ambient interaction region, with the plasma parameters detailed in Fig. 1 yielding $\beta_{\mathrm{e}} \sim 10^{-3}$. Finally, the debris ion-ambient ion and debris ion-ambient electron Coulomb collisional mean free paths of $\lambda_{i i} \sim 10 \mathrm{~km}$ and $\lambda_{i e} \sim 10 \mathrm{~m}$ significantly exceed the system size of $D \approx 50 \mathrm{~cm}$, confirming a collisionless interaction. The parameters thus ensure a regime in which laminar electric fields (for example, equation (1)) provide the dominant collisionless momentum exchange mechanism, allowing the laboratory environment to reproduce the key coupling physics of typical astrophysical and space explosions despite having orders-of-magnitude smaller spatial and temporal scales ${ }^{22}$. For comparison, one of the AMPTE barium release environments ${ }^{5}$ has $M_{\mathrm{A} \perp} \approx 4$ and $\beta_{\mathrm{e}} \sim 10^{-1}$ at the magnetic compression front, and the barium-solar wind Coulomb collisional mean free paths of $\lambda_{i i} \sim 10^{15} \mathrm{~km}$ and $\lambda_{i e} \sim 10^{13} \mathrm{~km}$ massively exceed the system size of $D \sim 10^{4} \mathrm{~km}$, similarly indicating a regime in which laminar coupling plays a significant role.

Direct evidence of Larmor coupling follows from emission spectroscopy, which detects large Doppler shifts in the $\mathrm{He}^{1+}$ ion $468.6 \mathrm{~nm}$ spectral line. As the laser-produced debris penetrates through the ambient plasma column, energetic electrons associated with $\mathbf{E} \times \mathbf{B}$ currents at the magnetic compression front excite ground-state $\mathrm{He}^{+1}$ ions and considerably intensify their 
a

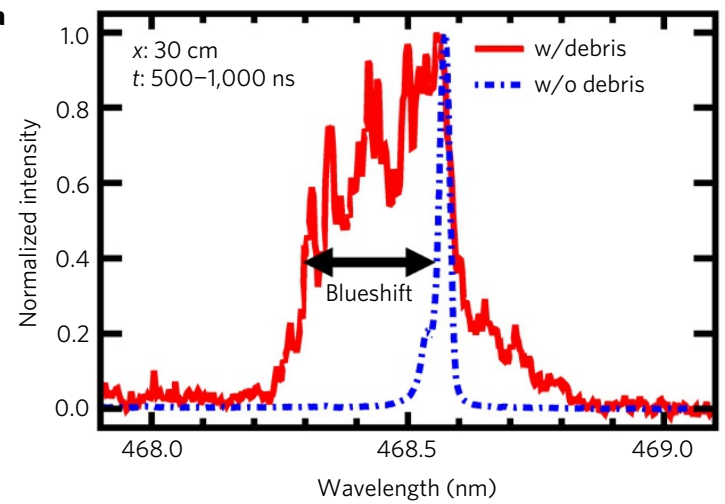

c

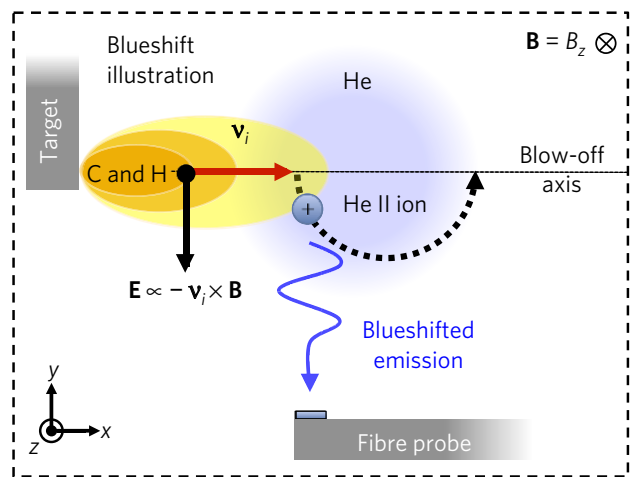

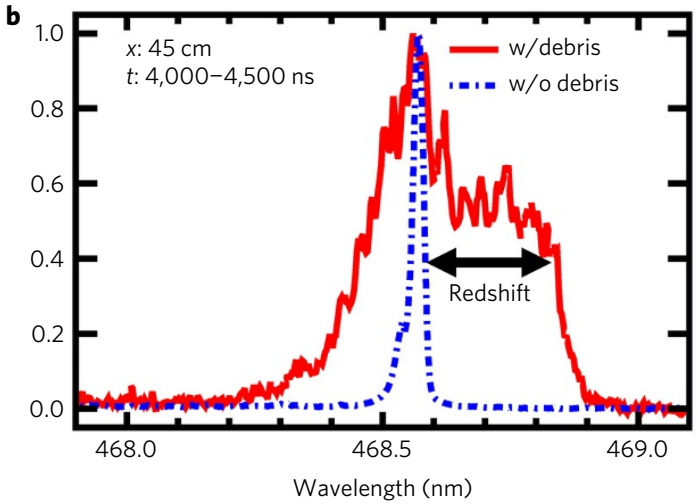

d

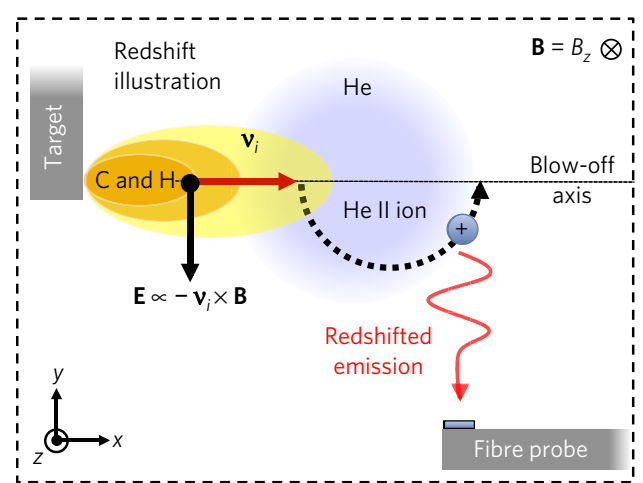

Figure 2 | Observation of Larmor coupling via Doppler shift. a, Normalized wavelength profiles of the $\mathrm{He}^{1+} 468.6 \mathrm{~nm}$ line measured with (solid red line) and without (dashed blue line) debris plasma at $30 \mathrm{~cm}$ from the target, time-integrated between $500 \mathrm{~ns}$ and 1,000 ns after the laser pulse. b, Wavelength

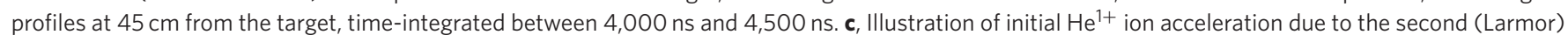
term of equation (1) towards the fibre probe, resulting in the Doppler blueshift detected in a. d, Illustration of the subsequent He ${ }^{1+}$ ion gyration in the magnetic field, resulting in the Doppler redshift detected in $\mathbf{b}$.

self-emission (see Fig. 1d). The spectroscopic fibre probe then samples the excited $\mathrm{He}^{+1}$ ions along the $y$ axis, perpendicular to both the primary debris flow direction and the magnetic field (see Fig. 1c). Fig. 2 shows normalized wavelength profiles measured at two different positions and time intervals, as well as corresponding illustrations that interpret the results. The first profile (Fig. 2a) is measured at $30 \mathrm{~cm}$ from the target and time-integrated from $500 \mathrm{~ns}$ to $1,000 \mathrm{~ns}$ after the laser pulse (the approximate interval during which the magnetic compression and diamagnetic cavity edge move through the fibre probe field of view), revealing a Doppler blueshift of $\Delta \lambda=-0.25 \pm 0.02 \mathrm{~nm}$ (at half-maximum) with respect to the analogous measurement without debris plasma. At the central transition wavelength of $\lambda_{c}=468.6 \mathrm{~nm}$, this corresponds to a directed velocity component along $y$ of $v_{y}=c \Delta \lambda / \lambda_{c}=-160 \pm 13 \mathrm{~km} \mathrm{~s}^{-1}$ towards the fibre probe, nearly two orders of magnitude faster than the thermal $\mathrm{He}^{1+}$ ion root-meansquare speed of approximately $3 \mathrm{~km} \mathrm{~s}^{-1}$ in the unperturbed ambient plasma. The second profile (Fig. $2 \mathrm{~b}$ ), measured at $45 \mathrm{~cm}$ from the target and time-integrated from $4,000 \mathrm{~ns}$ to $4,500 \mathrm{~ns}$, demonstrates a Doppler redshift of $\Delta \lambda=0.22 \pm 0.02 \mathrm{~nm}$ and corresponds to a directed velocity component along $y$ of $v_{y}=141 \pm 13 \mathrm{~km} \mathrm{~s}^{-1}$ away from the fibre probe, similar in magnitude but opposite in direction to the first measurement. The detected Doppler shifts are readily interpreted in terms of the laminar electric field $\mathbf{E}_{\text {lam }}$ of equation (1) and the coordinate system of Fig. 1. Along the blow-off axis, the various charge states comprising the debris plasma set up ion current densities $\sum_{i} Z_{i} n_{i} \mathbf{v}_{i}$ directed in $+x$ as they expand through the magnetic field $\mathbf{B}$ directed in $-z$, such that the second (Larmor) term of $\mathbf{E}_{\text {lam }}$ points in $-y$ (that is, the direction of $-\sum_{i} Z_{i} n_{i} \mathbf{v}_{i} \times \mathbf{B}$ ). As the debris cloud enters the ambient plasma column, the Larmor term of $\mathbf{E}_{\text {lam }}$ initially accelerates fluorescing $\mathrm{He}^{1+}$ ions located
$\left|B_{z}\right|(G)$

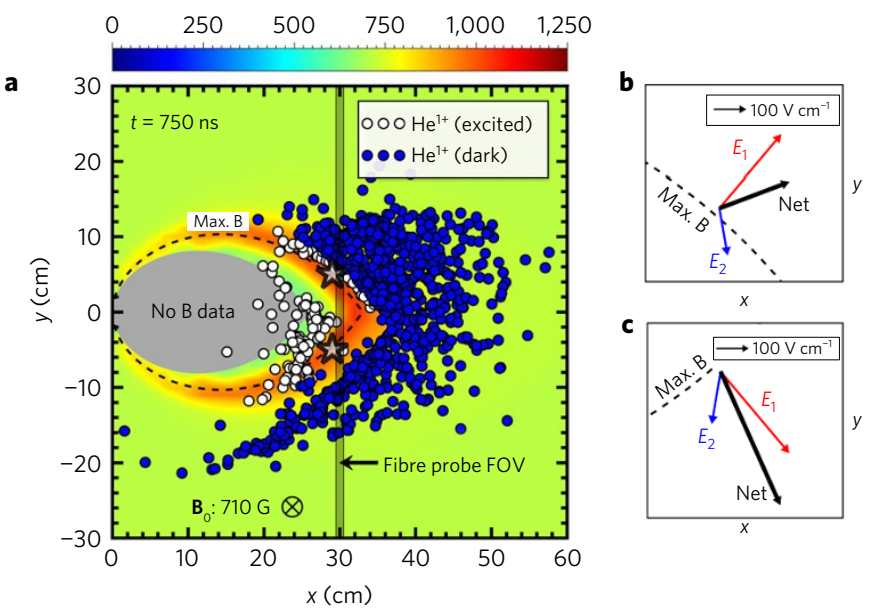

Figure 3 | Simulated $\mathrm{He}^{1+}$ test ion response. a, Simulated $\mathrm{He}^{1+}$ test ion positions in the blow-off plane at $750 \mathrm{~ns}$ after the laser pulse, superimposed on a colour contour of the magnitude of $\mathbf{B}$ (directed in $-z$ ). The excited test ions that contribute to the collected spectrum (white) as they pass through the fibre probe field of view at $x=30 \pm 0.5 \mathrm{~cm}$ are distinguished from the dark ions (blue) that do not contribute via planar images of $\mathrm{He}^{1+}$ ion excitation (for example, Fig. 1c). b,c, Relative contributions of the first (' $E_{1}$ ', red) and second (' $E_{2}$ ', blue) terms of equation (1) to the total $\mathbf{E}_{\text {lam }}$ ('net', black) at the two starred positions in $\mathbf{a}$, which are just ahead of the peak compression $\mathbf{B}$ contour (dashed line) at $(x, y)=(29,5) \mathrm{cm}$ (above the blow-off axis) and $(x, y)=(29,-5) \mathrm{cm}$ (below the blow-off axis), respectively. 

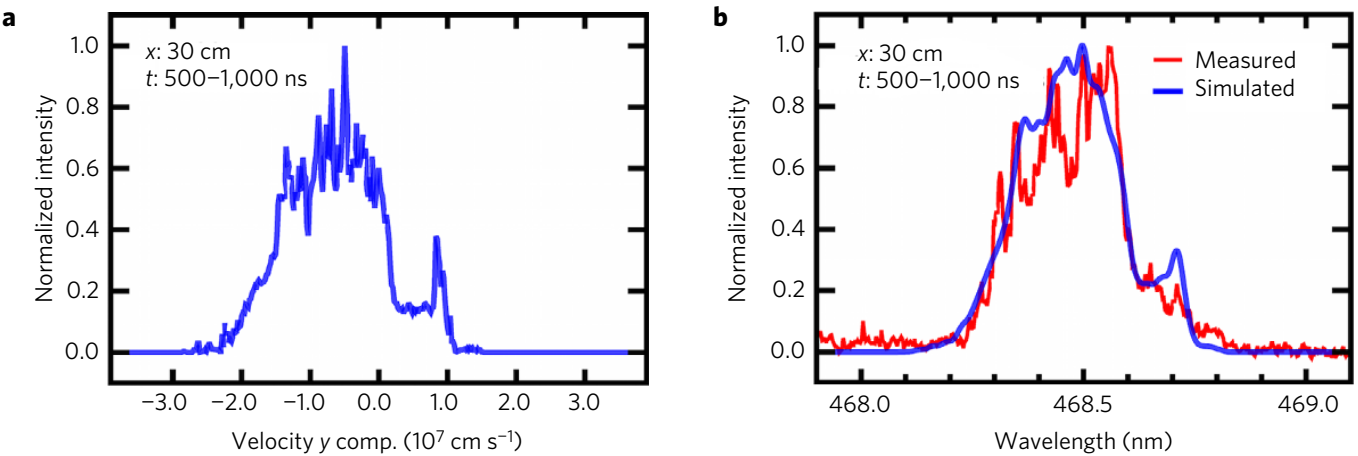

Figure 4 | Comparison of measured and simulated spectra. a, Simulated velocity y component distribution of the excited subset of test ions within the fibre probe field of view (see Fig. 3a) between 500 ns and 1,000 ns after the laser pulse. b, Corresponding synthetic wavelength spectrum of the He $\mathrm{e}^{1+}$ $468.6 \mathrm{~nm}$ line (blue) compared to the experimental measurement of Fig. 2a (red).

near the blow-off axis in the $-y$ direction and towards the fibre probe, producing the observed blueshift (Fig. 2c). The magnetic Lorentz force subsequently 'rotates' the accelerated ions into the $+y$ direction over a distance on the order of the directed gyro-radius (approximately $10 \mathrm{~cm}$ for $\mathrm{He}^{1+}$ ions with a speed of $160 \mathrm{~km} \mathrm{~s}^{-1}$ in the background magnetic field of $710 \mathrm{G}$ ), consistent with the redshift measured farther from the target and later in time (Fig. 2d).

The predominantly blueshifted spectral profile of Fig. 2a provides qualitative evidence that the second (Larmor) term of equation (1) participates in the initial acceleration of ambient $\mathrm{He}^{1+}$ ions. However, the simplified interpretation in terms of Fig. 2c does not account for the spatially and temporally integrated nature of the measurement or the complex $\mathrm{He}^{1+}$ ion trajectories resulting from the spatial and temporal dependence of the electric and magnetic fields, both of which contribute to the atypical $\mathrm{He}^{1+}$ ion velocity distribution represented by the Doppler-broadened spectrum. Moreover, the interpretation fails to explain the presence of a small redshift in Fig. 2a. To quantitatively evaluate whether the profile of Fig. 2a is indeed consistent with $\mathrm{He}^{1+}$ ion acceleration via the laminar electric field of equation (1), we employ a computational approach. Specifically, the response of a $\mathrm{He}^{1+}$ test ion distribution (initialized to match the experimentally measured ambient ion density and temperature profile of the LAPD) is computed in the blow-off plane via the Lorentz force $m_{\mathrm{a}} \mathrm{d} \mathbf{v}_{\mathrm{a}} / \mathrm{d} t=Z_{\mathrm{a}} e\left(\mathbf{E}_{\text {lam }}+\mathbf{v}_{\mathrm{a}} / c \times \mathbf{B}\right)$, where the electric field $\mathbf{E}_{\text {lam }}$ is explicitly evaluated via equation (1), the magnetic field $\mathbf{B}$ follows directly from the spatially and temporally resolved magnetic flux probe measurements, and $\mathbf{v}_{\mathrm{a}}, m_{\mathrm{a}}$ and $Z_{\mathrm{a}}=1$ are the velocity, mass and charge number of each ambient test ion. Selective sampling of the simulated test ion velocities then yields a synthetic Dopplerbroadened profile of the $\mathrm{He}^{1+} 468.6 \mathrm{~nm}$ line that is compared to the spectroscopic measurement of Fig. 2a.

To compute the spatial and temporal dependence of $\mathbf{E}_{\text {lam }}$ via equation (1) (see Supplementary Movie 1 for an animation of the calculated electric field), we develop a data-driven model of the laser-produced debris plasma generated in our experiment (see Supplementary Sections II and III for details). Specifically, filtered images of the debris cloud yield the expansion geometry (see Supplementary Fig. 1), while the experimentally validated radiationhydrodynamics code HELIOS ${ }^{29,30}$ predicts the debris ion charge state populations and velocity distributions expected for our laser pulse configuration (see Supplementary Table I). In evaluating $\mathbf{E}_{\text {lam }}$, we ignore the ambient ion motion and assume the debris plasma undergoes a radial, ballistic expansion. These simplifications are well justified during the relatively early time interval of the spectroscopic measurement (see Supplementary Section IV for details).

Figure $3 \mathrm{a}$ plots the simulated $\mathrm{He}^{1+}$ test ion response in the blowoff plane at $750 \mathrm{~ns}$ after the laser pulse, demonstrating that the ions are swept up and asymmetrically displaced with respect to the blow-off axis in the vicinity of the outwardly propagating magnetic compression (see Supplementary Movie 2 for an animation of the test ion response). The asymmetric push results from the structure of $\mathbf{E}_{\text {lam }}$ (equation (1)). In the marginally super-Alfvénic regime of the experiment $\left(M_{\mathrm{A} \perp} \approx 2\right)$, both the first (magnetic stress) and second (Larmor) terms contribute non-negligibly to the initial test ion response. In the blow-off plane, the first term contributes a radial-like outward component at the compression front while the second term points azimuthally 'clockwise' (see Supplementary Section II). As demonstrated in Fig. 3b,c, the vector sum of the two terms results in an asymmetric net $\mathbf{E}_{\text {lam }}$. Specifically, because typical magnitudes of the first term (approximately $300 \mathrm{~V} \mathrm{~cm}^{-1}$ at the sampled positions and time) exceed those of the second term (approximately $150 \mathrm{~V} \mathrm{~cm}^{-1}$ ), the net $\mathbf{E}_{\text {lam }}$ at the magnetic compression front is characterized by a small positive $y$ component in the upper half of the blow-off plane $(y>0)$ and a relatively large negative $y$ component in the lower half $(y<0)$. To mimic the spectroscopic measurement of Fig. 2a, the velocity y components of the excited subset of test ions located within the fibre probe field of view (see Fig. 3a) are sampled over the measurement time interval between $500 \mathrm{~ns}$ and 1,000 ns, yielding the velocity distribution shown in Fig. $4 \mathrm{a}$. The velocity distribution confirms that the asymmetrically structured $\mathbf{E}_{\text {lam }}$ accelerates test ions in the lower half of the blow-off plane to large negative 'blueshift' velocities along $y$ (magnitudes up to approximately $250 \mathrm{~km} \mathrm{~s}^{-1}$ ) and those in the upper half to comparatively small positive 'redshift' velocities (up to approximately $100 \mathrm{~km} \mathrm{~s}^{-1}$ ). Use of the standard Doppler relation $v_{y} / c=\Delta \lambda / \lambda_{c}$ and convolution with instrumental and fine structure broadening converts the test ion velocity distribution into the synthetic wavelength spectrum of the $\mathrm{He}^{1+} 468.6 \mathrm{~nm}$ line shown in Fig. 4b, which excellently reproduces the predominantly blueshifted and partially redshifted measured profile of Fig. 2a. The strong agreement provides quantitative evidence that both the first (magnetic stress) and second (Larmor) terms of equation (1) participate in $\mathrm{He}^{1+}$ ion acceleration and demonstrates that Larmor coupling ultimately accounts for the predominant blueshift in the measured spectrum.

We have reported the direct observation of Larmor coupling in a reproducible laboratory experiment that investigates the super-Alfvénic, quasi-perpendicular expansion of a laser-produced $\mathrm{C}$ and $\mathrm{H}$ debris plasma cloud through preformed, magnetized $\mathrm{He}$ ambient plasma. Doppler shifts in the $\mathrm{He}^{1+}$ ambient ion $468.6 \mathrm{~nm}$ spectral line indicate acceleration transverse to the debris flow and background magnetic field, and a detailed test ion simulation based on the laminar electric field of equation (1) successfully reproduces the measured spectrum, quantitatively confirming the participation of the Larmor process in the debrisambient interaction. The results of this study suggest that Larmor coupling explains the unanticipated findings of the AMPTE mission and participates in the evolution of a variety of space and 
astrophysical environments characterized by similar parameter regimes. Moreover, the impressive agreement between data and simulation achieved in this work provides a strong argument for the validity of the 'hybrid' approach, which has been utilized in a multitude of previous theoretical and computational investigations of explosive space phenomena. Finally, the experimental detection of Larmor coupling constitutes an important contribution to investigations of cosmic magnetized collisionless shock formation, in which this process plays a crucial role.

Data availability. The data that support the plots within this paper and other findings of this study are available from the corresponding author upon reasonable request.

Received 14 June 2016; accepted 16 January 2017; published online 20 February 2017

\section{References}

1. Haerendel, G., Paschmann, G., Baumjohann, W. \& Carlson, C. Dynamics of the AMPTE artificial comet. Nature 320, 720-723 (1986).

2. Valenzuela, A. et al. The AMPTE artificial comet experiments. Nature 320, 700-703 (1986).

3. Rees, D., Hallinan, T. J., Stenbaek-Nielsen, H. C., Mendillo, M. \& Baumgardner, J. Optical observations of the AMPTE artificial comet from the northern hemisphere. Nature 320, 704-708 (1986).

4. Eberhart, J. AMPTE's last blast: another comet made. Sci. News 128, 54 (1985).

5. Papadopoulos, K., Huba, J. \& Lui, A. Collisionless coupling in the AMPTE artificial comet. J. Geophys. Res. 92, 47-54 (1987).

6. Dunlop, M., Southwood, D. \& Mier-Jedrzejowicz, W. On a magnetic source of southward motion of the AMPTE solar wind barium release of 27 December 1984. Planet. Space Sci. 35, 493-500 (1987).

7. Brecht, S. H. \& Thomas, V. Three-dimensional simulation of an active magnetospheric release. J. Geophys. Res. 92, 2289-2304 (1987).

8. Bashurin, V., Golubev, A. \& Terekhin, V. The collisionless deceleration of an ionized cloud dispersing in a uniform plasma in a magnetic field. J. Appl. Mech. Tech. Phys. 24, 614-620 (1983).

9. Golubev, A., Solov'ev, A. \& Terekhin, V. Collisionless dispersion of an ionized cloud into a homogeneous magnetized plasma. J. Appl. Mech. Tech. Phys. 19, 602-609 (1978).

10. Spicer, D. S., Maran, S. P. \& Clark, R. W. A model of the pre-Sedov expansion phase of supernova remnant-ambient plasma coupling and $\mathrm{x}$-ray emission from SN 1987a. Astrophys. J. 356, 549-571 (1990).

11. Hewett, D. W., Brecht, S. H. \& Larson, D. J. The physics of ion decoupling in magnetized plasma expansions. J. Geophys. Res. 116, A11310 (2011).

12. Cargill, P. J. Collisions between quasi-parallel shocks. Adv. Space Res. 11, 241-244 (1991).

13. Draine, B. T. \& McKee, C. F. Theory of interstellar shocks. Annu. Rev. Astron. Astrophys. 31, 373-432 (1993)

14. Clark, S. E. et al. Hybrid simulation of shock formation for super-Alfvénic expansion of laser ablated debris through an ambient, magnetized plasma. Phys. Plasmas 20, 082129 (2013).

15. Mendis, D. \& Ip, W.-H. The ionospheres and plasma tails of comets. Space Sci. Rev. 20, 145-190 (1977).

16. Burlaga, L. F. et al. Fast ejecta during the ascending phase of solar cycle 23: ACE observations, 1998-1999. J. Geophys. Res. 106, 20957-20977 (2001).

17. Dyal, P. Particle and field measurements of the Starfish diamagnetic cavity. J. Geophys. Res. 111, A12211 (2006).
18. Wright, T. P. Early-time model of laser plasma expansion. Phys. Fluids 14, 1905-1910 (1971)

19. Papadopoulos, K. Heating of counterstreaming ion beams in an external magnetic field. Phys. Fluids 14, 849-857 (1971)

20. McBride, J. B., Ott, E., Boris, J. P. \& Orens, J. H. Theory and simulation of turbulent heating by the modified two-stream instability. Phys. Fluids 15, 2367-2383 (1972).

21. Berezin, Y. A., Dudnikova, G. I., Fedoruk, M. P. \& Vshivkov, V. A. Explosion phenomena in collisionless plasmas at super-Alfvénic speed. Int. J. Comput. Fluid Dynam. 10, 117-126 (1998).

22. Zakharov, Y. P. Collisionless laboratory astrophysics with lasers. IEEE Trans. Plasma Sci. 31, 1243-1251 (2003).

23. Niemann, C. et al. Observation of collisionless shocks in a large current-free laboratory plasma. Geophys. Res. Lett. 41, 7413-7418 (2014).

24. Schaeffer, D. B. et al. Laser-driven, magnetized quasi-perpendicular collisionless shocks on the Large Plasma Device. Phys. Plasmas 21, 056312 (2014)

25. Shaikhislamov, I. et al. Experimental study of collisionless super-Alfvénic interaction of interpenetrating plasma flows. Plasma Phys. Rep. 41, 399-407 (2015)

26. Bonde, J., Vincena, S. \& Gekelman, W. Electrostatic structure of a magnetized laser-produced plasma. Phys. Rev. E 92, 051102 (2015).

27. Gekelman, W. et al. The upgraded Large Plasma Device, a machine for studying frontier basic plasma physics. Rev. Sci. Instrum. 87, 025105 (2016).

28. Niemann, C. et al. High-energy Nd:glass laser facility for collisionless laboratory astrophysics. J. Instrum. 7, P03010 (2012).

29. MacFarlane, J., Golovkin, I. \& Woodruff, P. HELIOS-CR - a 1-D radiation-magnetohydrodynamics code with inline atomic kinetics modeling. J. Quant. Spectrosc. Radiat. Transfer 99, 381-397 (2006).

30. Schaeffer, D. et al. Characterization of laser-produced carbon plasmas relevant to laboratory astrophysics. J. Appl. Phys. 120, 043301 (2016).

\section{Acknowledgements}

We would like to thank the staff of the Large Plasma Device for their help in carrying out this experiment. This work was performed at the Basic Plasma Science Facility at UCLA, funded by the National Science Foundation (NSF) and the Department of Energy (DOE), and was supported by the Defense Threat Reduction Agency (DTRA) under contract number HDTRA1-12-1-0024, the DOE under contract numbers DE-SC0006538:0003 and DE-NA0001995, and NSF award 1414591.

\section{Author contributions}

All authors (except D.W.) participated in the infrastructure design, setup, and execution of the experiment. A.S.B. collected and analyzed the spectroscopic and imaging data, wrote the custom simulation code, generated the figures, and wrote the manuscript. D.B.S. designed and assembled the spectroscopic fiber probe, ran the HELIOS simulations, and developed the Raptor laser software. E.T.E. designed and assembled the magnetic flux probe and wrote the software routines to analyze the magnetic field data. S.E.C. assisted with interpretation of the data by utilizing hybrid code simulations. B.R.L collected and analyzed the magnetic field data. C.G.C. and C.N. designed, constructed, and operated the Raptor laser system. S.V., B.V.C. and S.K.P.T. configured and operated the LAPD and assisted with the installation of diagnostics and infrastructure. D.W. produced the original theoretical and computational work that led A.S.B. and C.N. to conceive the experiment.

\section{Additional information}

Supplementary information is available in the online version of the paper. Reprints and permissions information is available online at www.nature.com/reprints.

Correspondence and requests for materials should be addressed to A.S.B.

\section{Competing financial interests}

The authors declare no competing financial interests. 